\title{
In reply to the letter to the Editor, "HAQ score is an independent predictor of sustained remission in patients with rheumatoid arthritis"
}

\author{
Kyung-Eun Lee ${ }^{1} \cdot$ Shin-Seok Lee $^{1}(\mathbb{D}$
}

Received: 8 October 2017 / Accepted: 10 October 2017 / Published online: 17 October 2017

(C) Springer-Verlag GmbH Germany 2017

\section{Dear Editor,}

We greatly appreciate the thoughtful comments by the authors of the letter to the Editor on the statistical issue in our study [1]. As shown in Table 3 , all variables included in the univariate analysis had a $p$ value $<0.2$ and these were the values used in the multivariate models. We apologize for the omission in mentioning this. Univariate regressions were performed to identify the baseline individual variables and indices that may serve as predictors of sustained remission. While variables with a $p$ value $<0.05$ in either the Mann-Whitney $U$ test or the $\chi^{2}$ tests were shown in the univariate analysis, only those variables with a $p$ value $<0.2$ in the univariate analysis were subsequently entered stepwise into the multivariate model. Variables with significance levels $>0.1$ were removed, as they did not contribute to the model.

\section{Reference}

1. Lee KE, Choi SE, Xu H, Kang JH, Park DJ, Lee SS (2017) HAQ score is an independent predictor of sustained remission in patients with rheumatoid arthritis. Rheumatol Int. doi:10.1007/ s00296-017-3833-z

This reply refers to the comment available at doi:10.1007/ s00296-017-3849-4.

Shin-Seok Lee

shinseok@chonnam.ac.kr

Department of Rheumatology, Chonnam National University

Medical School and Hospital, 42 Jebong-ro, Dong-gu,

Gwangju 61469, Republic of Korea 